\title{
Joint Exploration and Analysis of High-Dimensional Design-Occupancy Templates
}

\author{
Muhammad Usman \\ York University \\ Toronto, Canada \\ usman@eecs.yorku.ca
}

\author{
Davide Schaumann \\ Rutgers University \\ Piscataway, USA \\ davide.schaumann@gmail.com
}

\author{
Brandon Haworth \\ York University \\ Toronto, Canada \\ brandon@eecs.yorku.ca
}

\author{
Mubbasir Kapadia \\ Rutgers University \\ Piscataway, USA \\ mubbasir.kapadia@gmail.com
}

Petros Faloutsos

York University, UHN-TRI

Toronto, Canada

pfal@eecs.yorku.ca

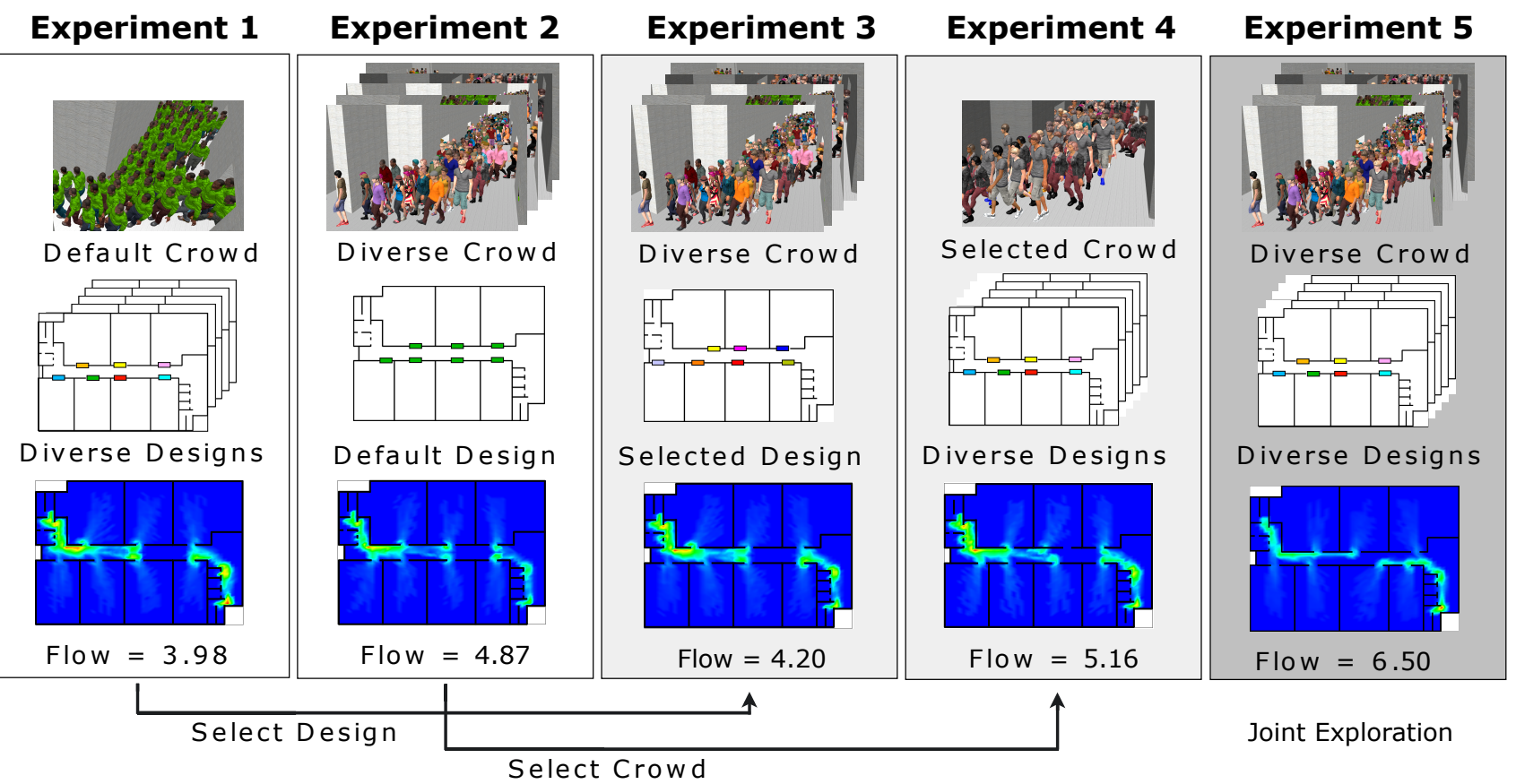

Figure 1: Experiment 1 explores diverse environment configurations using a default parameterized crowd, Experiment 2 explores a default parameterized environment against a diverse set of crowds, Experiment 3 explores the selected design from Experiment 1 using diverse crowds, Experiment 4 explores diverse environment configurations using the selected crowd from Experiment 2, and Experiment 5 jointly explores diverse environment and crowd configurations.

\author{
ABSTRACT \\ Crowd simulations provide a practical approach to evaluate build- \\ ing design alternatives with respect to human-centric criteria, such \\ Permission to make digital or hard copies of all or part of this work for personal or \\ classroom use is granted without fee provided that copies are not made or distributed \\ for profit or commercial advantage and that copies bear this notice and the full citation \\ on the first page. Copyrights for components of this work owned by others than ACM \\ must be honored. Abstracting with credit is permitted. To copy otherwise, or republish, \\ to post on servers or to redistribute to lists, requires prior specific permission and/or a \\ fee. Request permissions from permissions@acm.org. \\ MIG '19, October 28-30, 2019, Newcastle upon Tyne, United Kingdom \\ (c) 2019 Association for Computing Machinery. \\ ACM ISBN 978-1-4503-6994-7/19/10 ..\$15.00 \\ https://doi.org/10.1145/3359566.3360086
}

as evacuation times and flow in case of emergency scenarios. Coupled with Building Information Modeling (BIM) tools, they support architects' iterative exploration of design alternatives. However, methods based on manually configuring a design and a corresponding simulation are not practical for exploring the potentially very large number of design solutions that satisfy human-centric design goals and requirements. Often, for practical reasons, designers may consider standard crowd configurations which do not capture the behavior of diverse occupants that may exhibit different locomotion abilities, movement patterns, and social behaviors. We posit that a joint exploration of high-dimensional building design and occupancy features is necessary to more accurately capture the mutual relations between buildings and the behavior of their occupants. 
To test this hypothesis, we conducted a series of experiments to automatically explore joint high dimensional design-occupancy patterns using an unsupervised pattern recognition technique (i.e. K-MEANS). We demonstrate that joint design-occupancy explorations provide more accurate results compared with sequential exploration processes that consider default design or crowd features, despite the longer computational times to simulate a large number of solutions. The findings of this case study have practical applications to the design of next-generation design exploration tools that support human-centric analyses in architectural design.

\section{CCS CONCEPTS}

- Human-centered computing $\rightarrow$ Visual analytics; • Computing methodologies $\rightarrow$ Visual analytics; Classification and regression trees; Modeling and simulation; • Applied computing $\rightarrow$ Computer-aided design; Architecture (buildings).

\section{KEYWORDS}

Human-centric analytics, crowd simulation, parametric modeling, building occupancy, multi-agent systems.

\section{ACM Reference Format:}

Muhammad Usman, Davide Schaumann, Brandon Haworth, Mubbasir Kapadia, and Petros Faloutsos. 2019. Joint Exploration and Analysis of HighDimensional Design-Occupancy Templates. In Motion, Interaction and Games (MIG '19), October 28-30, 2019, Newcastle upon Tyne, United Kingdom. ACM, New York, NY, USA, 5 pages. https://doi.org/10.1145/3359566.3360086

\section{INTRODUCTION}

One of the major challenges in architectural design is the exploration of a wide range of design alternatives and the identification of those that best satisfy design goals while adhering to constraints [Kalay 2004]. Even though Building Information Modeling (BIM) tools support the analysis of physical aspects of buildings, such as structure, cost, lighting and energy consumption, at present, they cannot predict the impact that a building's design will produce on crowd movement. Synthetic crowd simulation is a costeffective means of exploring this space. However, crowd simulation approaches are mostly developed in the computer graphics community to represent the movement and dynamic interactions between virtual agents (e.g. Kapadia et al. [2015]). These approaches do not always account for the systematic impact that different building designs produce on occupant movement and activities. To capture the mutual relations between environment design and occupant behavior, it is necessary to study them jointly, in a shared modeling environment that enables the exploration of how environment features affect crowd behavior, and vice versa.

Prior work demonstrated a parametric modeling framework that jointly represents (a) a building and the bounds of its permissible layout alterations, (b) a crowd that populates the environment, and (c) the activities that the crowd engages in using a visualnode workflow commonly used in established parametric design approaches (e.g. Grasshopper [gra [n. d.]]) [Usman et al. 2019]. Users, such as architects or engineers, can manually adjust the building and crowd configurations, run crowd simulations, and analyze the results in the form of spatialized representations of human-centric analyses. This process, however, requires the manual exploration of a vast numbers of solutions in a high dimensional design space that uniquely combines space and crowd features.

In this paper, we conduct a series of experiments that use high value thresholding and an unsupervised pattern recognition technique to automatically explore the vast number of space-crowd parameter configurations. We posit that a joint exploration of highdimensional building design and occupancy features is necessary to comprehensively capture the mutual relations between buildings and the behavior of their occupants. To test this hypothesis, we systematically explore how design configurations and crowd features impact the flow of people in an evacuation scenario.

\section{RELATED WORK}

There is an established body of work exploring how crowd simulations can be incorporated in to architectural design processes to inform the design of human-centered environments.

\subsection{Crowd Simulations in Built Environments}

There is a rich literature on crowd simulation techniques to represent the movement of people in virtual environments [Kapadia et al. 2015; Pelechano et al. 2016]. Particle-based approaches compute local-level interactions among individuals for motion, speed and relative positions [Reynolds 1987]. Physical force models use repulsion and attraction to model individual crowd interactions [Helbing and Molnar 1995; Karamouzas et al. 2009].

\subsection{Simulating Human-Building Interactions}

Researchers have used crowd simulation techniques to simulate the movement of people in built environments to approximate buildingoccupant behaviors. Some approaches focus on day-to-day behavior of people in offices [Goldstein et al. 2010], hospitals [Schaumann et al. 2017], and universities [Shen et al. 2012]).

In these simulations, both crowd as well as building design factors affect the behavior of building occupants [Hong and Lee 2018; Hong et al. 2016]. Prior work has manually explored how different crowd configurations can affect the movement of people in environments [Chu et al. 2015]. Other studies have manually compared generated building design options to identify which design solutions better satisfy a set of key performance indicators [Schaumann et al. 2019]. Usman et al. [2018] presented an interactive architectural analysis tool to inform human-focused building designs. Interactive tools have been developed to study and evaluate the effects of pillar placements on crowd movement flows during evacuations [Haworth et al. 2017].

\subsection{Joint Exploration of Building Designs and Human-Behavior}

Recent work has focused on automatic user-in-the-loop generation of optimal building design solutions using dynamic crowd simulations [Feng et al. 2016; Haworth et al. 2017; Michalek and Papalambros 2002; Pottmann et al. 2015; Shi and Yang 2013; Turrin et al. 2011]. However, these methods are tailored to solve specific design problems, and are not integrated within established architectural design pipelines that include parametric building modeling. To address this, a system was recently proposed as a more comprehensive framework that jointly models (a) a building and the bounds 


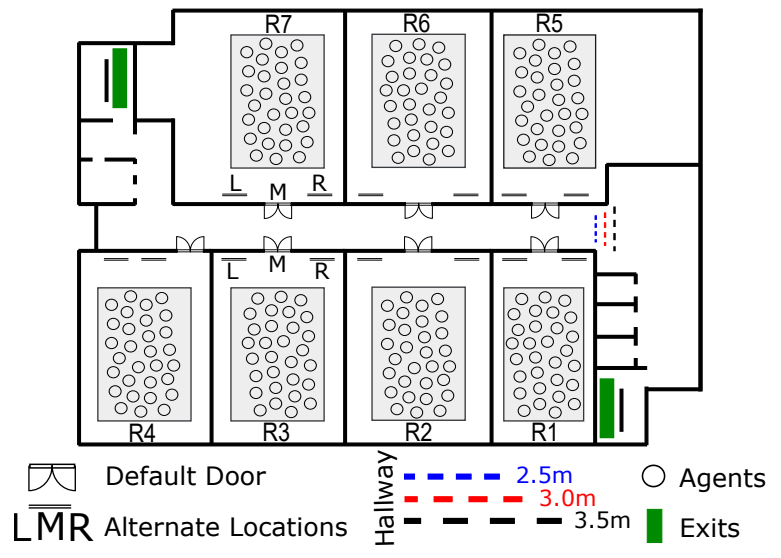

Figure 2: Design space of the environment used in case study experiments. GREEN regions are the exits. GRAY regions are the spawning areas for agents. BLACK solid lines are the boundary as well as interior walls. Default hallway (corridor) width is $3.5 \mathrm{~m}$. RED and BLUE dotted lines are hallway variants with $3.0 \mathrm{~m}$ and $2.5 \mathrm{~m}$ of width respectively. Openings in the walls facing the hallway are the default door placements. BLACK line segments parallel to the walls facing the hallway are the alternate locations for door placements.

of its permissible alterations, (b) the occupants that populate the building, and (c) the activities that people do on a day-to-day basis or in case of emergency scenarios Usman et al. [2019]. Designers can thus manually explore high-dimensional building-occupancy configurations and visualize the results in the form of spatialized data maps.

The aforementioned approaches, however, do not jointly model the parameter space of environment and crowd configurations, either treating them independently, or simplifying them. The focus of this study is to challenge this simplification, thereby showcasing that an automatic joint exploration of a vast number of solutions combining environment and crowd parameters is necessary to capture accurate building-occupancy relations and improve designs.

\section{EXPERIMENTAL SETUP}

We conduct a series of experiments to explore a large set of environment and crowd parameter configurations in "isolation" (i.e. environment/crowd only) as well as "jointly" to analyze crowd behaviors in a built environment for the said two workflows. Often, the design space exploration is based on environment parameters alone or at times using some constant crowd features (which may not exhibit different locomotion abilities and social behaviors) to account for human-behaviors for potential environment-occupant interactions. By doing so, designers may fail to identify a vast number of design solutions that satisfy human-centric environment design goals and requirements and may lead to ill posed designs that are unable to accurately capture the high dimensional design space.

The dimensionality of crowd and environment features is, taken individually, high and when considered jointly, multiplicatively large. As the feature dimensionality of either space grows the solution space grows rapidly and makes exhaustive exploration of solutions intractable-especially with continuous feature spaces. To address the exploration of such an enormous solution space, we adopt a method for finding patterns in high value designs of the exhaustive solution space. First, we discretize all solutions spaces regularly (in the system the granularity may be set by the user). Second, we threshold the highest value designs with respect to some given metric (in this paper we use crowds' exit flow) using only the 95th percentile of solutions (in the system the threshold value can be set by the user). Finally, we perform unsupervised pattern recognition to identify salient patterns in the remaining high value designs-we refer to these as templates. The templates are captured by clustering similar parameters (features) for environment and/or crowd configurations using an unsupervised pattern recognition technique, k-means++ with the squared Euclidean distance metric [Arthur and Vassilvitskii 2007; Hartigan and Wong 1979]. k-means++ uses a heuristic approach to seeding the cluster centroids and improves the quality of pattern solutions.

An environment template is the centroid of a cluster which represents the mean performance of all of the environment parameter configurations contained in that cluster with respect to the given performance criterion, i.e. Exit Flow. Similarly, a crowd template represents the mean performance of all the crowd parameter configurations in that cluster. The parameterized environment and crowd configurations are generated using a parametric design framework [Usman et al. 2019].

Exit Flow is used as a metric to evacuate simulations. It has been defined in several different ways [Helbing et al. 2007; Johansson et al. 2008]. Here, we define it as the rate at which the agents reach their goal or target position.

Table 1 shows an overview of the experimental setup. A total of five (5) experiments are conducted. First, we tested the sequential exploration approach by means of experiments (E1-E4). E1 explores the environment feature space using the default crowd parameterization. E2 explores the crowd feature space using the default environment parameterization. Using the environment templates found in E1, E3 explores the crowd features. Using the crowd templates found in E2, E4 explores the environment features. This experimental approach gives us the best of environment and crowd parameter templates from E4 \& E3 respectively. Finally, we explore the joint parameterization by testing all of the environment configurations using all of the crowd configurations (E5).

Figure 2 shows the default and alternate configurations of a real-world office space currently under construction. Table 2 and Table 3 show the environment and crowd parameters, and their values respectively.

\section{4 “SEQUENTIAL” VS “JOINT" PARAMETER EXPLORATION}

We simulate the best performing environment templates (Experiment 4) against the best performing crowd templates (Experiment 3) from the sequential parameter exploration process. Figure 3 (LEFT) shows the respective aggregate density maps from the sequential exploration process. Exit Flow value for the simulation is also reported. Environment template \# 1, simulated against crowd template \# 2, 
Table 1: An overview of the experimental setup. Further details on these experiments can be found in Section 3 . For experiments E1,E4 and E5, top 5\% of the flow-based sorted configurations (i.e. only the 95th percentile of solutions) are selected. Gray colored cells highlight the best performing of environment and crowd templates from sequential and joint parameter exploration respectively.

\begin{tabular}{|c|c|c|c|c|c|c|c|}
\hline $\begin{array}{c}\text { Experiments } \\
\text { (E) }\end{array}$ & $\begin{array}{c}\# \\
\text { Input } \\
\text { Environments } \\
\end{array}$ & $\begin{array}{c}\# \\
\text { Input } \\
\text { Crowds }\end{array}$ & $\begin{array}{c}\# \\
\text { Configurations } \\
\text { (Env. x Crowd) }\end{array}$ & $\begin{array}{c}\text { Top 5\% } \\
\text { Configurations } \\
\text { (Flow-based) }\end{array}$ & $\begin{array}{c}\text { \# Configurations } \\
\text { to } \\
\text { K-MEANS }\end{array}$ & $\begin{array}{c}\# \\
\text { Output } \\
\text { Environments }\end{array}$ & $\begin{array}{c}\# \\
\text { Output } \\
\text { Crowds }\end{array}$ \\
\hline $\begin{array}{c}\# \mathbf{1} \\
\text { Sequential } \\
\text { Environment }(\mathrm{v} 1)\end{array}$ & $\begin{array}{l}\text { Diverse } \\
(19,683)\end{array}$ & $\begin{array}{l}\text { Default } \\
\text { (1) }\end{array}$ & 19,683 & $\checkmark$ & 984 & $\begin{array}{c}\text { Salient } \\
\text { Environment } \\
\text { Templates (3) }\end{array}$ & \\
\hline $\begin{array}{c}\text { \# } 2 \\
\text { Sequential } \\
\text { Crowd (v1) }\end{array}$ & $\begin{array}{l}\text { Default } \\
\text { (1) }\end{array}$ & $\begin{array}{c}\text { Diverse } \\
(27)\end{array}$ & 27 & & 27 & & $\begin{array}{l}\text { Salient Crowd } \\
\text { Templates (2) }\end{array}$ \\
\hline $\begin{array}{c}\text { \# } 3 \\
\text { Sequential } \\
\text { Crowd (v2) }\end{array}$ & $\begin{array}{c}\text { Templates } \\
\text { from Exp \#1 } \\
\text { (3) }\end{array}$ & $\begin{array}{c}\text { Diverse } \\
(27)\end{array}$ & 81 & & 81 & & $\begin{array}{l}\text { Salient Crowd } \\
\text { Templates (2) }\end{array}$ \\
\hline $\begin{array}{c}\# \mathbf{4} \\
\text { Sequential } \\
\text { Environment (v2) }\end{array}$ & $\begin{array}{l}\text { Diverse } \\
(19,683)\end{array}$ & $\begin{array}{c}\text { Templates } \\
\text { from Exp \#2 } \\
\text { (2) }\end{array}$ & 39,366 & $\checkmark$ & 1,968 & $\begin{array}{c}\text { Salient } \\
\text { Environment } \\
\text { Templates (3) }\end{array}$ & \\
\hline $\begin{array}{c}\text { \# 5 } \\
\text { Joint } \\
\text { Environment/ } \\
\text { Crowd }\end{array}$ & $\begin{array}{l}\text { Diverse } \\
(19,683)\end{array}$ & $\begin{array}{c}\text { Diverse } \\
(27)\end{array}$ & 531,441 & $\checkmark$ & 26,572 & $\begin{array}{c}\text { Salient } \\
\text { Environment } \\
\text { Templates (3) }\end{array}$ & $\begin{array}{c}\text { Salient Crowd } \\
\text { Templates (3) }\end{array}$ \\
\hline
\end{tabular}

Table 2: Environment parameters and their permissible values. A default (D) environment configuration has design space parameters such that doors are placed in the middle, hallway width is $3.5 \mathrm{~m}$ and exits are on both (LEFT/RIGHT) sides of the environment.

\begin{tabular}{llll}
\hline \multicolumn{1}{c}{ Parameter } & \multicolumn{3}{c}{ Permissible Values } \\
\hline Door Placements & Left & Middle (D) & Right \\
\hline Hallway Width & $3.5 \mathrm{~m}$ (D) & $3.0 \mathrm{~m}$ & $2.5 \mathrm{~m}$ \\
\hline Exits & Left/Right (D) & Left only & Right only \\
\hline
\end{tabular}

yield the highest exit flow of 5.398 agents/second. Simulations from "sequential" exploration yield an exit flow of 4.80 agents/second on average. Also, the door placements are made such that they are opposite to each other expect for room 2 and 6, causing more congestion in the hallway, hence, slowing down the movements.

We also simulate the environment and crowd templates which were found from the joint parameter exploration process (Experiment 5). Figure 3 (RIGHT) shows the respective aggregate density maps from the joint exploration process. Exit Flow value for the simulation is also reported. Jointly discovered environment-crowd template \# 1 yield the highest exit flow of 6.488 agents/second. Simulations from joint exploration yield an exit flow of 5.762 agents/second on average. Parameterization from joint exploration produced $34.7 \%$ and $27.6 \%$ increased in exit flow compared to sequential best and
Table 3: Crowd parameters and their permissible values. A default (D) crowd configuration has crowd parameters such that occupancy is 200 agents, which are equally distributed within all seven rooms. In every room, agents are equally mapped to a set of three walking speeds, $1.0 \mathrm{~m} / \mathrm{s}, 1.3 \mathrm{~m} / \mathrm{s}$ and $1.6 \mathrm{~m} / \mathrm{s}$.

\begin{tabular}{|c|c|c|c|}
\hline Parameter & Perm & issible Values & \\
\hline Occupancy & 100 agents & 200 agents (D) & 300 agents \\
\hline $\begin{array}{l}\text { Agents } \\
\text { Distribution }\end{array}$ & Equal (D) & $\begin{array}{l}66 \% \text { equal } \\
33 \% \text { random }\end{array}$ & $\begin{array}{l}33 \% \text { equal } \\
66 \% \text { random }\end{array}$ \\
\hline $\begin{array}{l}\text { Speed } \\
(\text { meter/sec) }\end{array}$ & $\begin{array}{l}33 \% \text { agents }(1.0) \\
33 \% \text { agents }(1.3)(D) \\
33 \% \text { agents }(1.6)\end{array}$ & $\begin{array}{l}66 \%(1.0) \\
17 \%(1.3) \\
17 \%(1.6)\end{array}$ & $\begin{array}{l}17 \%(1.0) \\
17 \%(1.3) \\
66 \%(1.6)\end{array}$ \\
\hline
\end{tabular}

average simulation results respectively. Overall, joint exploration process produced better environment-crowd parameters which yield higher values for both highest and average exit flows. In addition, with joint parameter exploration, door placements are made such that they are offset to each other (i.e. not opposite), creating a sort of zipper effect in the crowd movements, hence, less congestion in the hallway and better exit flows. 


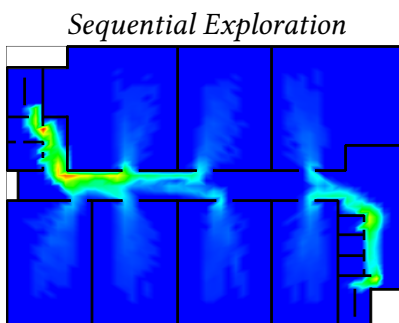

C2 | E1: Flow $=5.398$ agents $/$ s

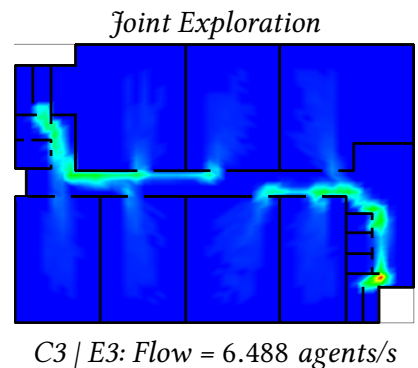

C3 | E3: Flow $=6.488$ agents $/ \mathrm{s}$
Figure 3: Density maps from sequential (LEFT) and joint (RIGHT) exploration of environment-crowd parameters during evacuations. Exit Flow values are also reported.

\section{CONCLUSION}

The presented work motivates the study of an automatic joint modeling of high-dimensional environment-crowd parameterization. The comparison indicates that joint exploration of environmentcrowd features (E5) yields higher value design solutions compared to sequential parameter explorations. In addition, the joint exploration produced environment layouts where doors are positioned offset to each other (as opposed to opposing doors found in the sequential parameter exploration, E3-E4), which resulted in smooth crowd movements and higher exit flows. However, in order for this to be a practical consideration, there is a significant computation overhead. Hence, future work is needed to expedite the computation process by means of machine learning and graphics acceleration techniques. Future work will also investigate individual parameters within environment and crowd configurations which have strong positive or negative impact with respect to design performance and the importance of generalized design features.

\section{ACKNOWLEDGMENTS}

This research has been partially funded by grants from the NSERC Discovery and Create programs, ISSUM and in part by NSF IIS1703883, NSF S\&AS-1723869 and the Murray Fellowship.

\section{REFERENCES}

[n. d.]. Grasshopper: Algorithmic Modeling for Rhino. https://www.grasshopper3d. com/

David Arthur and Sergei Vassilvitskii. 2007. k-means++: The advantages of careful seeding. In Proceedings of the eighteenth annual ACM-SIAM symposium on Discrete algorithms. Society for Industrial and Applied Mathematics, 1027-1035.

Mei Ling Chu, Paolo Parigi, Kincho H Law, and Jean-Claude Latombe. 2015. Simulating individual, group, and crowd behaviors in building egress. Simulation 91, 9 (2015), 825-845.

Tian Feng, Lap-Fai Yu, Sai-Kit Yeung, KangKang Yin, and Kun Zhou. 2016. Crowddriven Mid-scale Layout Design. ACM Trans. Graph. 35, 4, Article 132 (July 2016), 14 pages. https://doi.org/10.1145/2897824.2925894

Rhys Goldstein, Alex Tessier, and Azam Khan. 2010. Schedule-calibrated occupant behavior simulation. In Spring Simulation Multiconference. Society for Computer Simulation International, 180 .

John A Hartigan and Manchek A Wong. 1979. Algorithm AS 136: A k-means clustering algorithm. Journal of the Royal Statistical Society. Series C (Applied Statistics) 28, 1 (1979), 100-108.

Brandon Haworth, Muhammad Usman, Glen Berseth, Mahyar Khayatkhoei, Mubbasir Kapadia, and Petros Faloutsos. 2017. CODE: Crowd-optimized design of environments. CAVW (2017).

Dirk Helbing, Anders Johansson, and Habib Zein Al-Abideen. 2007. Dynamics of crowd disasters: An empirical study. Physical review E 75, 4 (2007), 046109.
Dirk Helbing and Peter Molnar. 1995. Social force model for pedestrian dynamics. Physical review E 51, 5 (1995), 4282.

Seung Wan Hong and Yun Gil Lee. 2018. The Effects of Human Behavior Simulation on Architecture Major Students' Fire Egress Planning. Fournal of Asian Architecture and Building Engineering 17, 1 (2018), 125-132.

Seung Wan Hong, Davide Schaumann, and Yehuda E. Kalay. 2016. Human behavior simulation in architectural design projects: An observational study in an academic course. Computers, Environment and Urban Systems 60 (Nov. 2016), 1-11.

Anders Johansson, Dirk Helbing, Habib Z Al-Abideen, and Salim Al-Bosta. 2008. From crowd dynamics to crowd safety: a video-based analysis. Advances in Complex Systems 11, 04 (2008), 497-527.

Yehuda E. Kalay. 2004. Architecture's new media: Principles, theories, and methods of computer-aided design. MIT Press, Cambridge, MA.

Mubbasir Kapadia, Nuria Pelechano, Jan Allbeck, and Norm Badler. 2015. Virtual Crowds: Steps Toward Behavioral Realism. Synthesis Lectures on Visual Computing 7, 4 (2015), 1-270.

Ioannis Karamouzas, Peter Heil, Pascal Van Beek, and Mark H. Overmars. 2009. A predictive collision avoidance model for pedestrian simulation. In Lecture Notes in Computer Science, Vol. 5884. Springer, 41-52.

Jeremy Michalek and Panos Papalambros. 2002. Interactive design optimization of architectural layouts. Engineering Optimization 34, 5 (2002), 485-501. https: //doi.org/10.1080/03052150214021

Nuria Pelechano, Jan M Allbeck, Mubbasir Kapadia, and Norman I Badler. 2016. Simulating Heterogeneous Crowds with Interactive Behaviors. CRC Press.

Helmut Pottmann, Michael Eigensatz, Amir Vaxman, and Johannes Wallner. 2015. Architectural geometry. Computers and Graphics (Pergamon) 47 (2015), 145-164. https://doi.org/10.1016/j.cag.2014.11.002

Craig W Reynolds. 1987. Flocks, herds and schools: A distributed behavioral model. SIGGRAPH 21, 4, 25-34.

Davide Schaumann, Simon Breslav, Rhys Goldstein, Azam Khan, and Yehuda E. Kalay. 2017. Simulating use scenarios in hospitals using multi-agent narratives. BPS 10, 5-6 (2017), 636-652.

Davide Schaumann, Nirit Putievsky Pilosof, Hadas Sopher, Jacob Yahav, and Yehuda E Kalay. 2019. Simulating multi-agent narratives for pre-occupancy evaluation of architectural designs. Automation in Construction 106 (2019), 102896.

Weilin Shen, Qiping Shen, and Quanbin Sun. 2012. Building Information Modelingbased user activity simulation and evaluation method for improving designer-user communications. Automation in Construction 21 (2012), 148-160.

Xing Shi and Wenjie Yang. 2013. Performance-driven architectural design and optimization technique from a perspective of architects. Automation in Construction 32 (2013), 125-135. https://doi.org/10.1016/j.autcon.2013.01.015

Michela Turrin, Peter Von Buelow, and Rudi Stouffs. 2011. Design explorations of performance driven geometry in architectural design using parametric modeling and genetic algorithms. Advanced Engineering Informatics 25, 4 (2011), 656-675. https://doi.org/10.1016/j.aei.2011.07.009

Muhammad Usman, Davide Schaumann, Brandon Haworth, Glen Berseth, Mubbasir Kapadia, and Petros Faloutsos. 2018. Interactive Spatial Analytics for Human-aware Building Design. In Motion, Interaction, and Games (MIG '18). ACM, New York, NY, USA, Article 13, 12 pages.

Muhammad Usman, Davide Schaumann, Brandon Haworth, Mubbasir Kapadia, and Petros Faloutsos. 2019. Joint Parametric Modeling of Buildings and Crowds for Human-Centric Simulation and Analysis. In Computer-Aided Architectural Design. "Hello, Culture”, Ji-Hyun Lee (Ed.). Springer Singapore, Singapore, 279-294. 